\title{
CFD Analysis of Impeller Trimming
}

\author{
Dušan Šlachtič ${ }^{1, *}$, Branislav Knížat ${ }^{1}$, and Róbert Olšiak $^{1}$ \\ ${ }^{1}$ Strojnícka Fakulta STU in Bratislava, Nám. slobody 17, 81231 Bratislava, Slovak Republic
}

\begin{abstract}
Impeller trimming is a key impeller modification. It allows the best efficiency point (BEP) to be moved towards the region of lower flowrate and height, thus optimizing pump performance for specific piping systems. The paper deals with prediction of trimming results using CFD methods. Experience shows that modification of the BEP position depends on the trimming method as well as on the pump type and its specific speed. The analysed pump is of diagonal type with a specific speed of $n_{b}=0.168$. Its impeller is of a 4-blade design and a spiral casing is used as a volute. Seven cases of trimming are presented (including a non-trimmed original version). The paper compares CFD obtained data and data measured on an experimental stand. Additionally, the approach to CFD analysis, as well as the use of a turbulence model and characteristics of internal pump volume meshing are described.
\end{abstract}

\section{Introduction}

In order to keep manufacturing costs low, every pump manufacturer tries to cover the biggest possible region in a Q-Y (Flowrate-Specific energy) chart with a minimal number of manufactured pump types. One possible way of achieving this goal is by trimming the impeller of a pump. The diameter of the original impeller is gradually reduced, thus creating a pump with different $\mathrm{Q}-\mathrm{Y}$ characteristics and with a new best efficiency point (BEP) location. Impeller trimming also affects the overall efficiency of pumps. This process is known to be applied for fan modifications as well [1]. While the physical background of changing the BEP point is the same, each pump type (according to its specific speed $n_{b}$ ) is trimmed differently based on manufacturers` experience. The simplest form of trimming is applied to pumps with a radial impeller as the trailing edge is located on a cylindrical surface. In such a case, the trimming results depend on a simple change of the impeller outer diameter $D_{2}$. Trimming becomes more complex when applied to diagonal and axial impellers, where the trimmed trailing edge lies on a conical surface. As a result, effective trimming depends not only on the impeller outer diameter, but on the cone angle too. Some authors have also examined the influence of the trailing edge shape [2].

There are multiple papers about creating a mathematical model usable for trimming results prediction, focussing mainly on the prediction of the BEP location and also the efficiency value at this point. Numerous approaches to create such a model exist, the simpler of which relate to the validity of affinity equations for trimming an impeller. However, this assumption has not proven to be valid, namely because modification of the impeller diameter

\footnotetext{
*Corresponding author: dusan.slachtic@stuba.sk
} 
does not preserve its geometrical similarity [3]. Theoretical analysis of diameter change effect dependent on outlet angle $\beta_{2}$ is described in a paper by Tsang [4]. A limiting factor of this approach, which comes from single dimensional flow theory, is knowledge of the finite blade correction factor. There is a wide disparity in the value of this correction factor according to the calculation method used. Based on knowledge gained from experiments, the dependence of flowrate and the specific energy of the trimmed impeller is represented as a simple power function $\left(D_{2}^{\prime} / D_{2}\right)^{n}$, where $D_{2}^{\prime}$ is the trimmed diameter and $D_{2}$ is the original, non-trimmed diameter. The power $n$ depends on the type of pump (its specific speed) and is usually determined experimentally. The value of power $n$ is different for both flowrate and specific energy evaluation.

A significant tool contributing to make parameter change prediction of trimmed impellers more accurate is the method of computational fluid dynamics (CFD) simulation [1, 5]. This method is helpful in the development of trimming stages as it allows the user to change all geometric parameters including trailing edge shape. It is possible to obtain optimal location of BEP while maintaining the best possible efficiency value. This paper analyses the use of CFD simulation in a case of a trimmed mixed flow impeller and a spiral casing volute. Results obtained by the simulation are compared to the ones measured experimentally.

\section{Impeller and volute geometry}

Impeller trimming is very complex one and mastering it requires experimental approach too. There is no way of making reliable predictions without validation of trimmed impeller calculated parameters.

Table 1. Impeller trimmings.

\begin{tabular}{|c|c|c|}
\hline Trimming & $\mathbf{D}_{2}(\mathbf{m m})$ & Ratio (-) \\
\hline $\mathrm{t}_{0}$ & 243 & 1 \\
\hline $\mathrm{t}_{1}$ & 239.92 & 0.984 \\
\hline $\mathrm{t}_{2}$ & 229.82 & 0.946 \\
\hline $\mathrm{t}_{3}$ & 223.18 & 0.918 \\
\hline $\mathrm{t}_{4}$ & 215 & 0.885 \\
\hline $\mathrm{t}_{5}$ & 205 & 0.844 \\
\hline $\mathrm{t}_{6}$ & 197 & 0.810 \\
\hline
\end{tabular}

The specific speed of the analysed impeller was $n_{b}=0.168$ and its original, non-trimmed diameter was $243 \mathrm{~mm}$. This version is referenced as $t_{0}$ in Table 1, whereas the trimming stages are referenced as $t_{1}$ to $t_{6}$. The pump consists of a four-bladed semi-open impeller made of sheet steel, which provides blades uniform thickness. These blades are bent into helical shape and welded onto the back disc of the impeller. Figure 1 shows a section view of the pump, and Figure 2 shows a photo of the impeller. The scheme of trimming stages is shown in Figure 3 and it can be seen that the trailing edge shape changes. Cases $t_{0}$ and $t_{1}$ utilize a trailing edge consisting of two lines, while other cases utilize simpler shape. Cases $t_{2}$ and $t_{3}$ feature a conical shape and cases $t_{4}$ to $t_{6}$ feature a traditional cylindrical trailing edge. Testing was performed at a constant pump rotational speed of $1500 \mathrm{~min}^{-1}$ and clearance between the stator and the impeller blades was set to $0.1 \mathrm{~mm}$ for all measurement cases. 


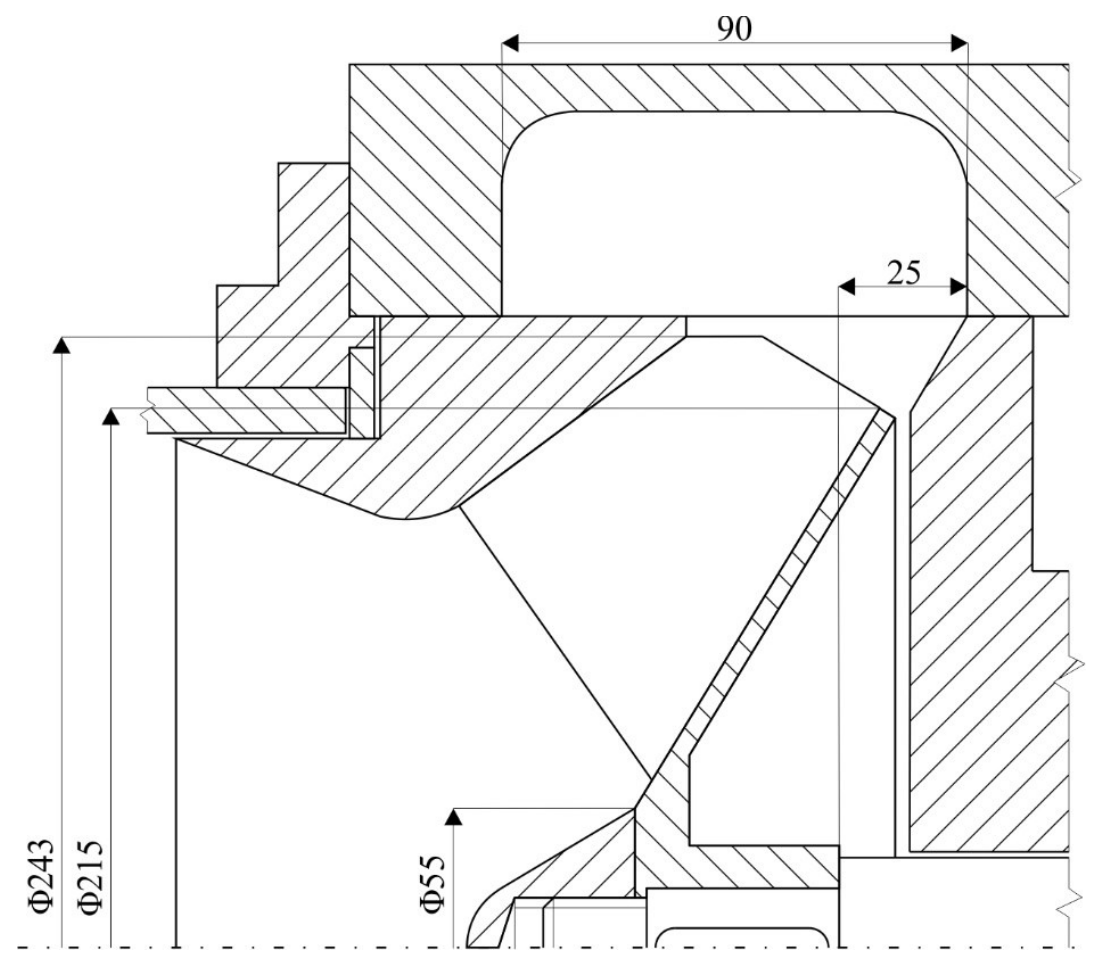

Fig. 1. Pump assembly section view (impeller and volute).

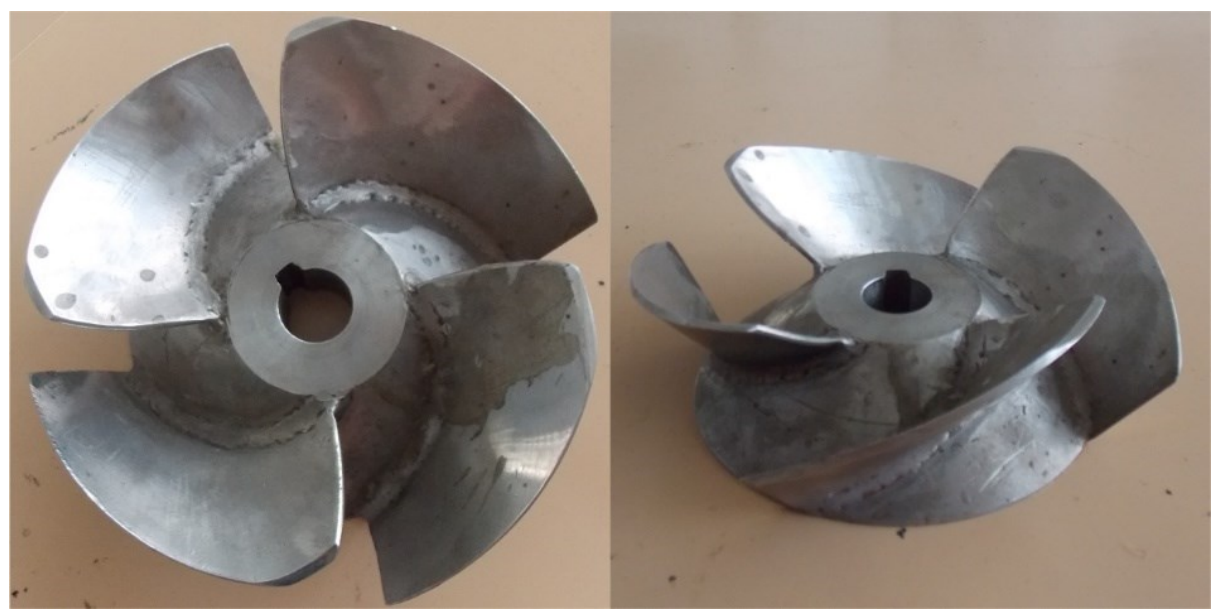

Fig. 2. Picture of the trimmed impeller.

The volute of the pump was a plastic spiral casing with an outlet diameter of $150 \mathrm{~mm}$. The relative position of the impeller and the spiral casing is shown in Figure 1.

All measurements were executed on a hydraulic open loop and physical quantities needed for evaluation of performance parameters such as flowrate, specific energy, input power and efficiency were measured. 


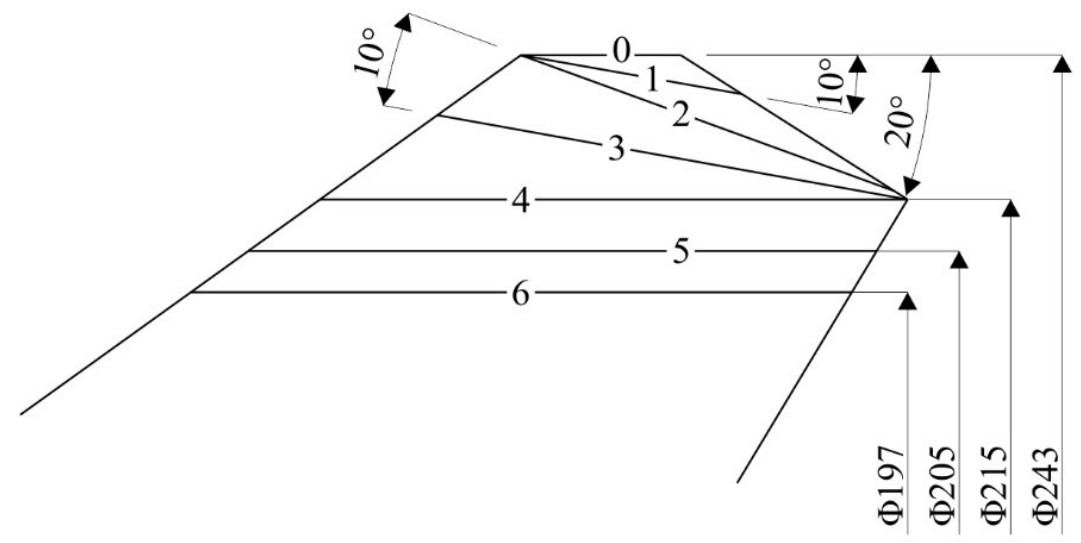

Fig. 3. Geometry of trimming cases.

\section{CFD simulation}

Flow simulations of the operating pump were executed using a Fluid flow CFX module contained by ANSYS software suite version 2019 R2. Firstly, a 3D model of a pump cavity was created and then inserted into ANSYS software. This model consisted of 3 domains that can be differentiated according to their function: namely, the inlet pipe, the impeller and the volute. The next step in the simulation process is called meshing. In each of the three main domains a fine mesh of elements was created in order to allow the simulation to run. During this part of the process, the volume of the domains is divided into a finite number of small regions in which the calculation is performed. It can be said that the simpler the shapes of the created regions are, the better for calculation. The meshing procedure was carried out using an ANSYS Meshing module and Table 2. Shows the number of elements for each domain for the case $t_{0}$. Every case featured mesh with a similar element number. Figure 4 shows a section of a mesh for case $t_{0}$. The boundary layer at the walls is represented by the first layer of mesh, $5.10^{-5} \mathrm{~m}$ thick.

Table 2. Numbers of mesh elements for case $t_{0}$.

\begin{tabular}{|c|c|}
\hline Domain & Elements \\
\hline Inlet pipe & 463914 \\
\hline Impeller & 4682987 \\
\hline Volute & 5231626 \\
\hline Altogether & 10378527 \\
\hline
\end{tabular}

The flow analysis type was set as Steady State. For each fluid domain a specific turbulent model must be chosen for the calculation. In this case, the Baseline Explicit Algebraic Reynolds Stress Model (BSL EARSM) was selected for fluid domains and Stage (Mixingplane) was selected for interfaces between each of the domains [6]. The Inlet pipe and Volute domains were set as stationary, whereas the Impeller domain had a rotational speed of 1500 revolutions per minute, which is the same as the real pump featured in the test. Boundary conditions were set so that both the inlet and outlet pressure was atmospheric and the inlet mass flowrate was set at the beginning of each run to obtain performance characteristics for different flowrate values. The process of simulation was repeated for each case from $t_{0}$ to $t_{6}$ and the results are analysed in the next chapter. 


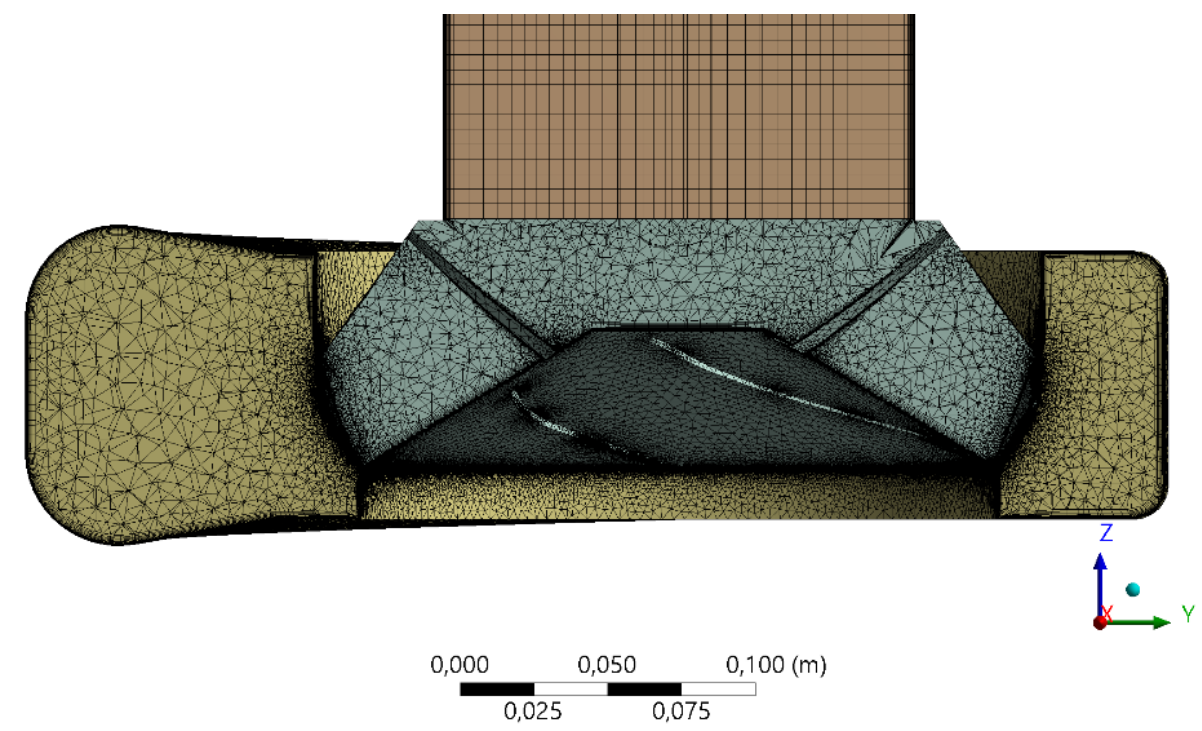

Fig. 4. Mesh section for case $t_{0}$.

\section{Results of investigations}

A change of performance parameters was monitored when analysing the effect of impeller trimming. Both the set of measurement obtained data and the set of data obtained by CFD simulation were compared.

Figure 5 is a graph with experimentally obtained results. Performance curves and constant efficiency curves are featured. All 7 cases from non-trimmed $t_{0}$ to maximally trimmed case $t_{6}$ are shown. The BEP point of each curve is the same as the point of maximum efficiency marked as $\eta_{\max }$, and it changes with each trimming stage.

The results of the CFD simulation are only relevant in a region close to the BEP point as the simulations were executed with steady state analysis. Figure 6 features a comparison of measured and simulated positions of maximum efficiency points for each trimming stage. There is a visible continual decrease of flowrate and specific energy with the decrease of the impeller diameter. Measured and simulated data do not overlap completely. It is supposed that this spread is caused by manufacturing imperfections, as the impeller blades were welded onto its back disc. It can however be said that the compliance of experiment and simulation is good. The graph plotted in Figure 6 shows that the CFD methodology along with the meshing quality and the turbulence model were chosen correctly. Simulation results positioned far from the BEP point cannot be compared to the ones obtained experimentally as the steady state analysis was set. Figure 7 demonstrates another significant result which can be used as a baseline for future analyses. 


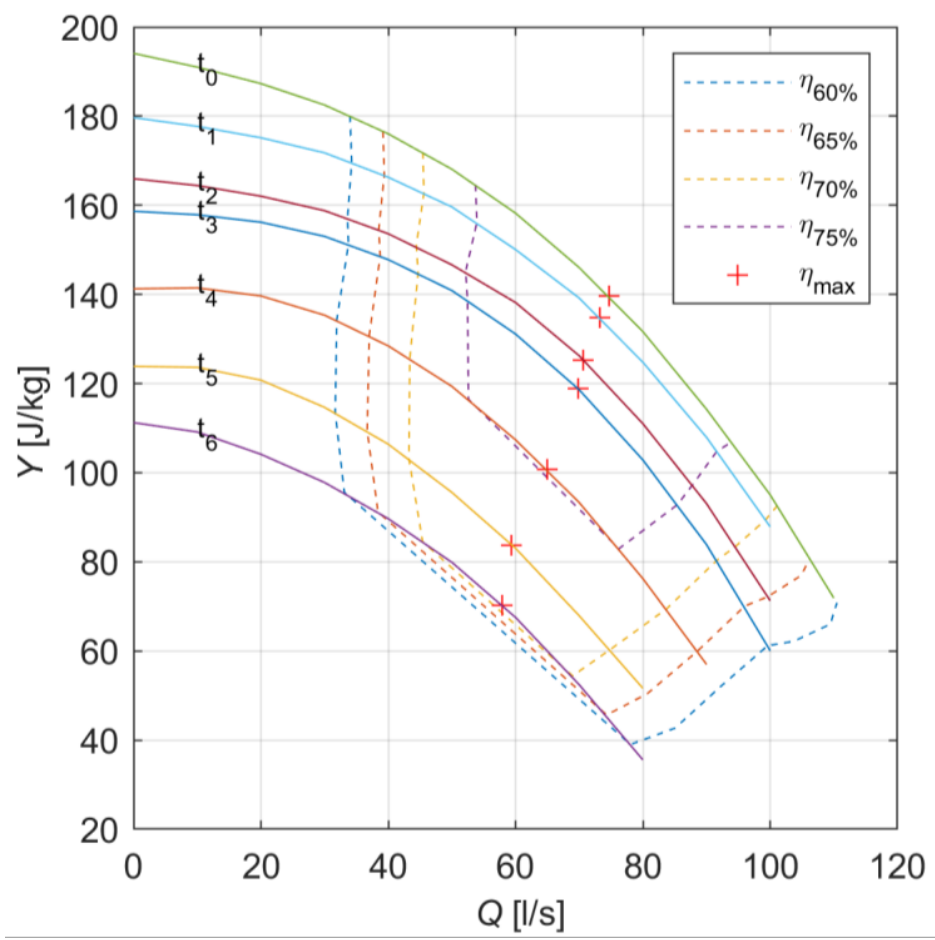

Fig. 5. Measured performance curves.

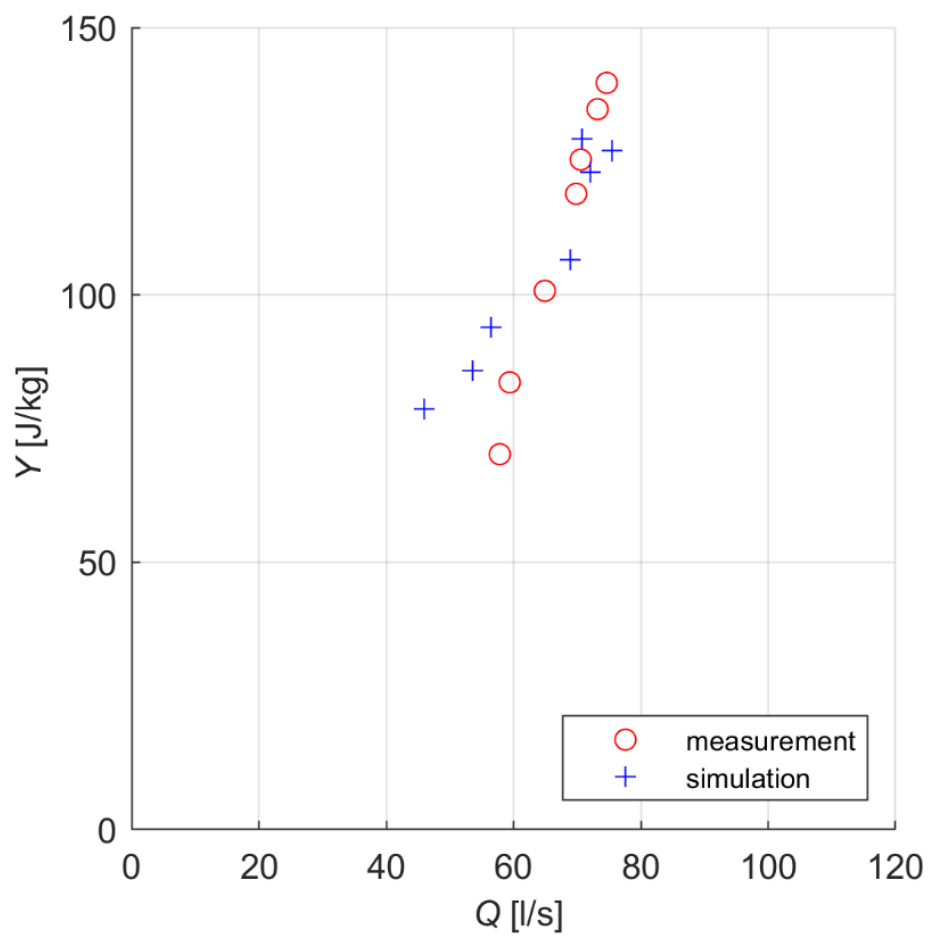

Fig. 6. Positions of the BEP for all trimming cases. 
Figure 7 represents the dependence of optimal parameter values: flowrate $Q$, specific energy $Y$ and maximum efficiency $\eta_{\max }$ on relative trimming level $\left(D_{2}^{\prime} / D_{2}\right)$.

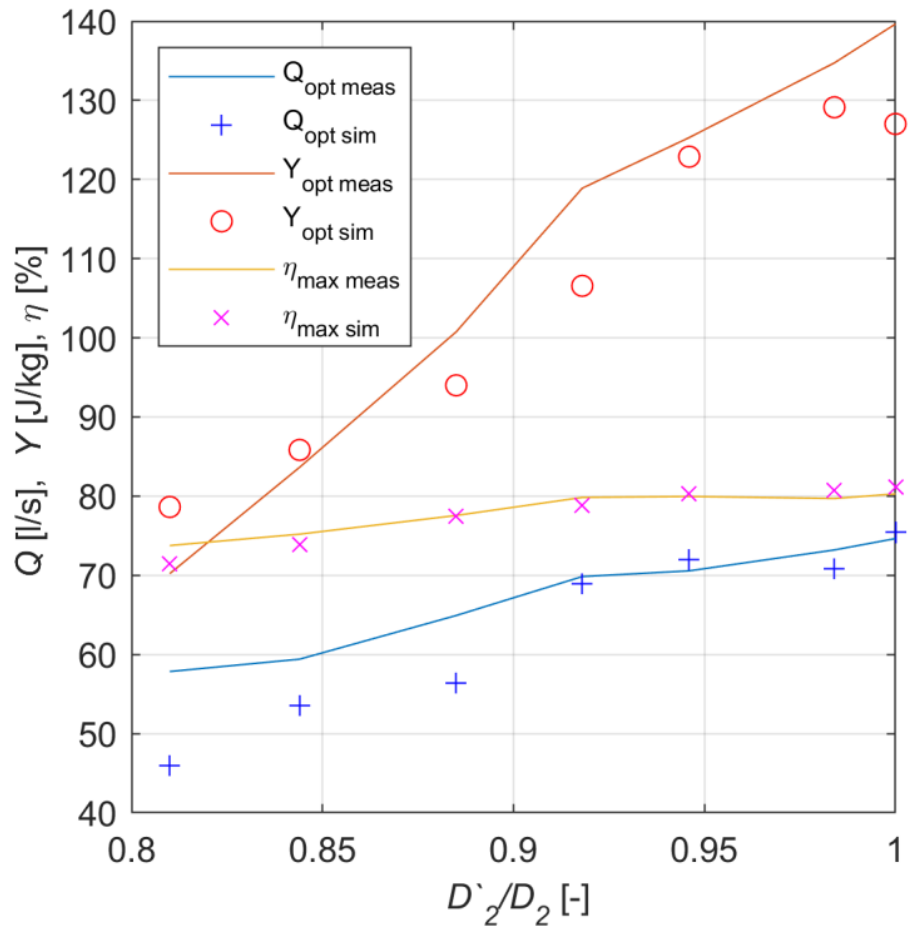

Fig. 7. Dependence of flow rate, specific energy and efficiency on trimming ratio.

Results obtained by measurement are represented by lines, and results obtained by simulation are represented by points. Both measured and simulation-obtained data show the same trend of the trimming effect. With the growing value of relative trimming level $\left(D^{\prime}{ }_{2} / D_{2}\right)$, the rise in flowrate, specific energy and also efficiency occurs. In the region of low relative trimming level, there is a discrepancy between the measured and simulated flowrates, which can be caused by the aforementioned manufacturing imperfections of the impeller. There is, however, no problem to obtain power functions to predict further trimming results.

\section{Conclusion}

This paper shows results of impeller trimming research applied to a change of parameters of a 4-bladed, mixed flow impeller. The key aspect is the determination of the BEP point position change represented by flowrate, specific energy and value of maximum efficiency achieved by CFD simulation. The results obtained by CFD simulations are comparable to the ones obtained by experimental measurement. The chosen pump used a steel impeller with uniform thickness blades welded onto an impeller back disc. The untrimmed impeller diameter was $D_{2}=243 \mathrm{~mm}$. This diameter was gradually modified in 6 stages. The final trimming stage diameter was $81 \%$ of the original diameter. A comparison of measured and simulated performance values in the BEP point was taken into account. Settings used for CFD simulation of flow in the specific pump can be assumed to be suitable, as compared results compliance is good. 
The described approach to CFD simulation is a suitable method of BEP point prediction for trimmed mixed flow impeller and blade modifications.

\section{References}

1. C. Li, X. Li, P. Li, X. Ye, Energy 75534 (2014)

2. D. Khoeini, M. R. Tavakoli, FME Transactions 46463 (2018)

3. M. Šavar, H. Kozmar, I. Sutlović, Desalination 249654 (2009)

4. L. M. Tsang, Proc Instn Mech Engrs. 206213 (1992)

5. Y. Sun-Sheng, K. Fan-Yu, J. Wan-Ming, Q. Xiao-Yun, Computers \& Fluids 6772 (2012)

6. M. Kurilla, B. Knížat, R. Olšiak, P. Slovák, CFD Analysis of Flow in a Single-blade Impeller, AIP Conference Proceedings 2118030023 (2019)

Acknowledgement
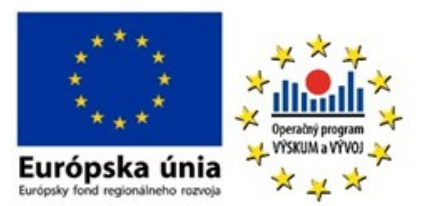

This contribution was created on the basis of the project "Research centre ALLEGRO" (ITMS project code: 26220220198), supported by Operational Programme Research and Development funded by the European Regional Development Fund.

The authors gratefully acknowledge the contribution of the Scientific Grant Agency of the Slovak Republic under the grant VEGA 1/0743/18. 